\title{
Seguiniento al año y 8 años de cohortes de alcohólicos tratados con disulfiram oral e implantable
}

\author{
González, R.**; Donaire, I. * *, Goicolea, S.**; Martínez, N.* ** \\ * Profesor Jefe del Servicio Rogelio Paredes Hospital Psiquiátrico Habana. **Psiquiatras del Servicio Rogelio Paredes H.P.H. \\ ***Trabajadora Social del Servicio Rogelio Paredes \\ Enviar correspondencia a: \\ Dr Ricardo González. Hospital Psiquiátrico de la Habana. Ave de Independencia 26520 Boyeros. C Habana Cuba CP 19220.
}

\section{RESUMEN:}

El seguimiento mediante visita domiciliaria por estudiantes de medicina de 5 to año a sendos grupos de dependientes alcohólicos primarios después de l y 8 años del egreso hospitalario en que recibieron un tratamiento integral al que se sumó el disulfiram implantable u oral como recurso disuasivo añadido, evidenció que el grupo de implantados (A) triplicó en el primer corte y duplicó en el segundo el porcentaje de pacientes abstinentes así como la valoración integral evolutiva de excelente alcanzados en el grupo B. Se demostró además, que los resultados excelentes fueron similarmente consistentes entre un corte y el otro en ambos grupos.. El número de fallecidos del grupo oral (B), triplicó al del grupo implantado (A). y la edad promedio de muerte fue de 42,8 años, cifra muy inferior a la expectativa de vida del hombre en Cuba, que supera los 74 años. Los resultados recomiendan mantener el disulfiram implantable como recurso disuasivo suplementario-.

Palabras Clave: Alcoholismo; tratamiento, disulfiram oral, disulfiram implantable.

\begin{abstract}
:
Follow up by house visits made by fifth year medical students to each group of primary alcoholic dependants, 1 year and 8 years after hospital discharge during which they received an integral treatment which included implantable and oral disulphiram as an added dissuasive recourse, showed that the implanted group (A) tripled on the first visit and duplicated on the second the percentage of abstinent patients, in addition to the overall evolutionary evaluation of excellence achieved in Group B. It was also shown that the excellent results were similarly consistent between the first and second visit in both groups. The number of deaths in the oral group (B), tripled that of the implanted group $(A)$ and the average age of death was 42.8 years, a very much lower figure general male life expectancy in Cuba which is more than 74 years. The results recommend implantable disulphiram as a supplementary dissuasive recourse
\end{abstract}

Key words: alcoholism, treatment, oral disulphiram, implantable disulphiram

\section{INTRODUCCIÓN}

$\mathbf{L}$ a efectividad del disulfiram en programas terapéuticos integrales para alcohólicos, ha sido mundialmente avalada (Kaplan et al 1998), y a diferencia de la proyección aversiva durante las primeras décadas de su utilización, la tendencia actual es a su indicación como recurso disuasivo(Galanter et al 1997) (Ciraulo et al 1991), es decir, para apoyar la decisión de abstinencia y no para establecer acondicionamientos de rechazo al alcohol (Rente et al 1989)

La evidencia clínica de frecuentes tácticas para neutralizar sus efectos en el caso de pacientes con actitu- des ambivalentes ante la ingestión de alcohol, determinó que se ensayaran fórmulas que lograran una acción mas prolongada que la alcanzada mediante su administración oral y surgieron así las formas inyectables de larga duración y las de implantación subcutánea.

Los resultados de un estudio de seguimiento por 1 año efectuado en nuestro medio (González et al 1995), apuntaron hacia mejores resultados con la modalidad de implante subcutáneo en región periumbilical. No existen sin embargo experiencias que nos permitan contribuir a dar respuesta al problema investigativo enunciable como ¿son estos resultados igualmente esperables a plazos más prolongados. 


\section{OBJETIVOS}

\section{Generales}

Obtener elementos para valorar la utilidad comparativa a largo plazo, del disulfiram oral e implantable con fines disuasivos en el contexto de un programa terapéutico integral. para pacientes alcohólicos que requirieron hospitalización.

\section{Específicos}

1 Determinar los patrones de consumo etílico y la proporción de pacientes abstinentes al año y 8 años después del egreso

2 Establecer la evolución en cada etapa, según los criterios del equipo y de las fuentes indirectas exploradas

3 Delimitar la consistencia evolutiva de cada paciente en las etapas valoradas

\section{MATERIALY MÉTODO.}

Todos los dependientes alcohólicos primarios entre 20 y 50 años, sin otras afecciones asociadas, hospitalizados en el servicio Rogelio Paredes del Hospital Psiquiátrico de la Habana durante la etapa comprendida entre el 31 de diciembre de 1990 y el 1 de enero de 1991 fueron incluidos, mediante tabla de números aleatorios en el grupo $A$ que recibió disulfiram implantable, o en el grupo $B$ que tomó disulfiram oral, ambos en el contexto de un tratamiento integral descrito detalladamente en publicaciones anteriores (González. 1993).

Luego de obtenerse el consentimiento escrito para participar en el estudio, la dosis oral del bloqueador metabólico fue de $500 \mathrm{mg}$ durante 5 días y después de $250 \mathrm{mg}$ durante 6 meses, en tanto que los implantados recibieron 5 pelets de $100 \mathrm{mg}$ en región paraumbilical. El seguimiento fue efectuado después de 1 y 8 años del egreso, mediante visita domiciliaria por estudiantes de 5to año de medicina luego de haber sido seminariados para aplicar una encuesta orientada a obtener información de un familiar adulto conviviste durante los últimos 10 años con el paciente y un miembro de la directiva del Comité de Defensa de la Revolución de su cuadra (CDR). Las fuentes indirectas fueron preferidas por su mayor confiabilidad demostrada en estudios anteriores (González et al 1992)

Durante su hospitalización los pacientes participaron en un programa integral que incluyó psicoterapia individual y de grupo así como terapia ocupacional en el marco de una comunidad terapéutica con un sistema de gratificación por fichas (token economy).

En los casos fallecidos y en los no localizados se produjo además la visita al terreno por parte de la trabajadores social del servicio. Los patrones de consumo de los fallecidos se recogieron hasta el momento de su deceso, aunque la evolución se consideró siempre como mala cuando la causa de muerte se vinculó al alcoholismo .La tabulación de las encuestas se produjo sin que se conociera el grupo de pertenencia y los criterios exigidos para la valoración integral evolutiva fueron:

Evolución excelente 1- Dejó la bebida total y permanentemente, 2- Las dos fuentes lo consideran mejorado, 3- No repercusión laboral familiar ni social, 4- No complicaciones somáticas ni psíquicas

Evolución Buena 1- Bebe esporádicamente pero sin excesos, 2-Las dos fuentes lo consideran mejorado, 3- No repercusión laboral ni social, 4- No complicaciones somáticas ni psíquicas.

Evolución Regular 1-Bebe en forma excesiva, pero no ha tenido nuevos tratamientos, 2-Ninguna de las fuentes lo considera mejorado, 3-No repercusión social, 4- Pueden existir complicaciones somáticas o psíquicas.

Evolución Mala 1-Bebe en forma excesiva y ha recibido nuevos tratamientos, 2- Ninguna de las fuentes lo considera mejorado o igual, 3-Hay repercusión familiar, laboral y social, 4- Hay repercusiones somáticas o psíquicas.

Todos los pacientes implantados recibieron la información de que la actividad del implante se mantendría de uno y medio a dos años.

\section{RESULTADOS}

La mayor proporción de los integrantes de los grupos presentó al momento del egreso, edades comprendidas entre 26 y 43 años $(67,1 \%$ del grupo A y $66 \%$ del grupo B) y la edad promedio en igual orden respectivo fue de 37,4 y 33,1 años; el color de la piel predominante fue el blanco con 63,7 y 58\%--propor- 
ción similar a la presente en la población general--; el 30 y $34 \%$ reportó mantener pareja estable; el 46 y $44 \%$ reportó escolaridad superior a secundaria; el 80 y 64\% salarios entre 101 y 300 pesos; el consumo excesivo de alcohol alcanzó promedios de 7,1 y 6,6 años y la bebida de mas frecuente consumo fue el ron o el aguardiente en el 82 y $80 \%$ de los casos. No se evidenciaron diferencias con significación estadística en los aspectos referidos.

De los 60 pacientes incluidos en el grupo A (Implantados) y los 53 del grupo B (Oral), se localizaron en el primer corte 58 y 50 para el 96,6 y $94,3 \%$ respectivamente y en el segundo 55 y 48 para el 91,7 y $90,6 \%$ en igual orden. Las causas de no localización fueron: 4 por cambios de residencia a otras provincias y 1 por salida del país en el grupo A, y 2 por direcciones no localizables, 2 por permutas a otras provincias y 1 por salida del país en el grupo B. El primer corte evolutivo se efectuó como promedio al I,3 años y el segundo a los 8,4.

El porcentaje de abstinentes en los grupos A y B fue respectivamente de 51,7 y 24 en el primer corte y de 32,7 y 20,8 en el segundo, mientras que los reportados como consumidores "normales" alcanzaron en igual orden respectivo 17,2 y 14 en el primer control y de 20 y $25 \%$ en el segundo.

\section{TABLA 1}

\begin{tabular}{|c|c|c|c|c|}
\hline \multicolumn{5}{|c|}{$\begin{array}{l}\text { VALORACION EVOLUTIVA INTEGRAL EN EL PRIMERO } \\
\text { Y SEGUNDO CORTES }\end{array}$} \\
\hline & \multicolumn{2}{|c|}{ Primer corte } & \multicolumn{2}{|c|}{ Segundo corte } \\
\hline & (GA) & $\%$ (GB) & (GA & $\%(G B)$ \\
\hline Excelente & 50 & 16 & 25,4 & 12,5 \\
\hline Buena & 13,7 & 8 & 20 & 14,6 \\
\hline Regular & 6,1 & 12 & 27,3 & 18,7 \\
\hline Mala & & 64 & 27,3 & 54,2 \\
\hline
\end{tabular}

\section{TABLA 2}

\begin{tabular}{|c|c|c|c|c|}
\hline \multicolumn{5}{|c|}{$\begin{array}{l}\text { VALORACION EVOLUTIVA SEGÚN LAS FUENTES } \\
\text { INDIRECTAS EXPLORADAS }\end{array}$} \\
\hline & \multicolumn{2}{|c|}{ Primer corte } & \multicolumn{2}{|c|}{ Segundo corte } \\
\hline & (GA) & $\%(\mathrm{~GB})$ & (GA) & $\%$ (GB) \\
\hline Mejor & 63,7 & 26 & 43,6 & 29,2 \\
\hline Igual & 7 & 14 & 25,4 & 20,8 \\
\hline Peor & 29,2 & 60 & 30, & 50. \\
\hline
\end{tabular}

\section{TABLA 3}

\begin{tabular}{|c|c|c|c|c|cc|c|cc|c|c|}
\hline \multicolumn{7}{|c|}{ CONCORDANCIA EVOLUTIVA ENTRE EL PRIMEROY YEGUNDO CORTES } \\
A PARTIR DE LA PRIMERA EVALUACIÓN \\
\hline Ealorados (GA)(GB)Valorados(GA) (GB) Valorados (GA)(GB) Valorados(GA) (GB) \\
\hline Excelente & $\%$ & Buena & $\%$ & Regular & $\%$ & Mala & $\%$ \\
\hline EB & 48 & 50 & BE & 0 & 25 & RE & 0 & 16,6 & ME & 0 & 0 \\
\hline ER & 20 & 25 & BB & 14,3 & 50 & RB & 33,3 & 0 & MB & 18,7 & 10 \\
\hline EM & 14 & 12,5 & BM & 57 & 0 & RR & 0 & 33,3 & MR & 37,5 & 20 \\
\hline
\end{tabular}

Nota: los casos no localizados después del primer corte habían sido evaluados como: Grupo A 1 B y 2 M; Grupo B 2 M.

La tabla 1 expone el porcentaje de pacientes en cada categoría evolutiva. Apréciese que la valoración de Excelente en el grupo implantado triplicó la del grupo B en el primer corte y la duplicó en el segundo, mientras que la categoría Mala fue duplicada--en ambos cortes-- por el grupo B con relación al A. En la tabla 2 se aprecia la concordancia del criterio de las fuentes familiares con relación a la valoración evolutiva del equipo y en la tabla 3 se expone el porcentaje de congruencia entre las evaluaciones de ambos cortes. Nótese la consistencia similar en ambos grupos en los pacientes considerados de evolución excelente en la primera evaluación, lo que a grandes rasgos plantea que después de esa valoración, aproximadamente la mitad mantuvo igual curso; la cuarta parte pasó a la categoría de evolución Buena y la otra cuarta parte se distribuyó entre regular y mala.

Debe destacarse además, que en el grupo A se produjeron dos fallecimientos (l por Infarto del miocardio a los 42 años y I por cirrosis hepática a los 40) mientras que en el grupo $B$ ocurrieron 6 muertes ( 4 por infarto del miocardio en edades respectivas de 42 , 38, 42, 40 años; I por bronconeumonía 56 años y 1 por Cáncer de pulmón 43 años).

\section{DISCUSIÓNY CONCLUSIONES}

Los resultados apuntan hacia una mayor efectividad del disulfiram implantable utilizado en el contexto de un tratamiento integral y se ratifican en el segundo corte las tendencias evidenciadas luego del primer año del egreso (González et al 1995). Las cifras de abstinencia finales fueron inferiores al 35\% reportado por (Mendoza 1989) y (Renté 1989) en estudios anteriores en nuestro servicio utilizando el disulfiram con 
propósitos aversivos, pero debe considerarse que el seguimiento fue solamente de un año. Por otra parte resultaron muy superiores a las evidenciadas en el seguimiento de 5 y 12 años de una cohorte de pacientes tratados en un contexto integral similar, pero con la ipecacuana como recurso aversivo (González et al 1999). Fueron también mayores que el $15 \%$ de abstinentes reportado por Helzer en seguimiento de 2 años (Heltzer 1985).

Se ratificó además, la tendencia internacional a que el infarto del miocardio desplace a la cirrosis hepática como primera causa de muerte en alcohólicos, y la edad promedio de los fallecidos que solo alcanzó 42,8 años, resultó muy cercana al promedio de 40,2 años registrado en un estudio sobre complicaciones del alcoholismo en nuestro medio en otra cohorte (González et al. 2000), cifra significativa en un medio donde la expectativa de vida en los hombres alcanza los 74 años.

Finalmente, consideramos que aun cuando los efectos del disulfiram implantado no sobrepasan los 18 meses, el mantenimiento durante ese tiempo de un estilo de vida seco, parece explicar las ventajas evolutivas constatadas, toda vez que los pacientes fueron informados de la duración de la implantación, con lo que se excluye el mecanismo placebo. Por otra parte, la mayoría de los tratados por vía oral interrumpieron el tratamiento antes del primer corte.

Concluimos destacando que en los resultados del presente estudio, el disulfiram implantable resultó de mayor efectividad al lograr mayor porcentaje de abstinentes, mejor evolución en el criterio del equipo y de las fuentes exploradas, así como menor mortalidad y resultados consistentes luego de agotarse el tiempo de actividad del fármaco bloqueador.

\section{AGRADECIMIENTOS.}

A los alumnos de 5 to año de Medicina, Facultad Universitaria Calixto García, Usbail Santana, Frank Caravia, Johandra Calixto, Harold Noa, Frank Vargas, que han colaborado activamente en esta investigación.

\section{BIBLIOGRAFÍA}

Ciraulo D.; Renner J. Alcoholism in Clinical Manual of Chemical Dependence Ciraulo D.; Shader R. Editors American Psychiatric Press Washington 1991.

Galanter M.; Kleber H. Tratamiento de los trastornos por abuso de substancias. Edit. Masson Barcelona 1997.

González R.; González M.; Calzadilla L.; Cisneros A. Los cuestionarios breves en la identificación de alcohólicos y bebedores sociales: Estudio comparativo entre la fuente directa y familiar. Rev. Del Hosp. Psiq. de la Hab.13(2) 97-106, 1992.

González R El Alcoholismo y su atención específica. Editorial Ciencias Médicas. La Habana 1993.

González R.; Martínez M.;Vázquez V.; Bacelo R. Estudio comparativo del disulfiram oral e implantable como recursos disuasivos antialcohólicos. Rev del Hosp Psiq. de la Hab36(1)1-6,1995

González R.; Goicolea S.; Calzadilla L.;Martínez H. El dependiente alcohólico a los 5 y 12 años de su egreso. Adicciones 11(3)279-282,1999.

González R.; Goicolea S.; Calzadilla L.; Martínez H. La repercusión del alcoholismo en una cohorte de pacientes seguidos a los 5 y 12 años después del egreso. Revista Española de Drogodependencias 25 (1) 49-56, 2000.

Helzer J. The extend of long term moderate drinking among alcoholics discharged from medical and psychiatric treatment facilities. The New England Journal of Medicine June 27, pag 1678-1682,1985.

Kaplan, H.; Sadock, B .Synopsis of Psychiatry. Williams and Wilkins Editors Baltimore 1998

Mendoza L. El disulfiram y el estímulo eléctrico aversivo: su valoración como recursos terapéuticos en el alcoholismo. Tesis de Grado La Habana 1989.

Rente S.; González R. La ipecacuana y el Disulfiram Su valoración como recursos aversivos en el Tratamiento del Alcoholismo Rev del Hosp Psiq Hab, 31(2),257 a 266,1989 . 